\title{
White Blood Cells and the Diagnosis of $\alpha$-Glucosidase Deficiency
}

\author{
J. C. DREYFUS ${ }^{(17)}$ AND L. POENARU \\ Institut de Pathologie Moléculaire, I.N.S.E.R.M. U 129, 75674 Paris Cedex 14, France
}

\section{Summary}

Leukocytes were obtained from 14 healthy subjects, one patient with the infantile form, two patients with the adult variant of acid maltase deficiency, two patients with chronic myelocytic leukemia, two patients with acute myeloid leukemia, and two patients with chronic lymphotic leukemia. In addition, lymphocytes were prepared from three normal subjects, and five established lymphoid lines were used.

Cells were extracted either with Triton $0,2 \%$, or with water followed by $0.2 \%$ Triton. $\alpha$-Glucosidase activity was measured in water homogenates, water extracts after centrifugation, and Triton extracts, with or without antisera directed against acid maltase (EC 3.2.1.3) and renal maltase (EC 3.2.1.20). The percentage of acid and renal maltases was then calculated in each soluble fraction.

Normal whole leukocytes (mostly granulocytes) contain both acid and "renal" maltases, whereas normal lymphocytes contain very little or no "renal maltase." This isozyme is present in chronic myelocytic leukemia, but is absent in acute myeloid and chronic lymphocytic leukemia as well as in established lymphoid lines.

Acid maltase is almost completely extracted with water, whereas renal maltase is extracted only with Triton. From the results, it appears that for the diagnosis of alpha glucosidase deficiency, cells should be extracted in water and centrifuged before determination.

Lymphocytes, which are devoid of renal maltase, are a better diagnostic material than are granulocytes.

\section{Speculation}

A specific isozyme of $\alpha$-glucosidase, renal maltase, is found in the organism only in kidneys and white blood cells, whereas all other tested organs, including fibroblasts and cultured amniotic cells, do not possess this isozyme (unpublished results). Because its extractability differs from that of acid maltase, this property can be taken advantage of in activity determinations. The "renal" isozyme is still present in leukemic myelocytes, but not in myeloblasts. It seems, therefore, to be absent in young cells as well as in all cells of lymphocytic origin.

The diagnosis of the various forms of $\alpha$-glucosidase deficiency is generally made on muscle biopsies or cultured fibroblasts. Urine has been proposed but shows wide physiologic variations. Acid $\alpha$-glucosidase is a lysosomal enzyme, and in most cases, the diagnosis of lysosomal diseases is made on white blood cells. Many authors have attempted to use these cells for $\alpha$-glucosidase deficiency with variable results $(1-4,9,10,12-14)$. It has been claimed that lymphocytes are a better source than are whole white blood cells (15).

We have recently demonstrated (7) that white blood cells possess a specific isozyme of $\alpha$-glucosidase, which is immunologically identical to the renal maltase isolated by de Burlet and Sudaka (5). This observation explains the finding of a residual activity in white blood cells, as well as in kidney, in patients with Pompe's disease or with the late form of the deficiency. This type of maltase shows kinetic differences from both acid and neutral ubiquitous maltases. It also possesses some characteristic properties of extractibility. Using antisera obtained against acid and renal $\alpha$-glucosidases, we have reinvestigated the possibility of using white blood cells for the study of acid maltase deficiency.

\section{MATERIALS AND METHODS}

\section{MATERIALS}

White blood cells were prepared by sedimentation in dextrane, followed by lysis of red cells by $0.17 \mathrm{M}$ ammonium chloride (11). Lymphocytes were isolated by sedimentation in a Ficoll-Hypaque gradient as described (11).

Leukocytes were obtained from 14 healthy subjects, one patient with the infantile form, and two patients with the adult variant of acid deficiency. White blood cells were also obtained from leukemic patients (chronic myelocytic, chronic lymphocytic, and acute myeloblastic leukemias). Lymphoid cultures, obtained from white blood cells infected by Epstein-Barr virus (8) were kindly donated by $G$. Lenoir (IARC, Lyon, France).

Antirenal maltase antiserum was prepared against pure human renal maltase (5) and was a gift of Drs. de Burlet and Sudaka, Faculté de Médecine, Nice, France).

Anti-acid maltase antiserum, prepared against human liver acid maltase, was a gift of Dr. Tager (Jansen Institute, Amsterdam, Netherlands).

\section{METHODS}

White blood cells were extracted by two techniques: (1) distilled water, by freezing and thawing five times; (2) $0.2 \%$ Triton X-100.

In typical experiments, the leukocyte pellet was extracted first by water and centrifuged at $10,000 \times g$ for $10 \mathrm{~min}$. The pellet was washed with water and then extracted by Triton. The various fractions were then incubated with an excess of antiserum and left overnight at $4^{\circ} \mathrm{C}$. Polyethylene glycol was then added to $5 \%$ concentration.

After centrifuging $(5000 \times \mathrm{g}, 10 \mathrm{~min}), \alpha$-glucosidase activity was determined at 4.0 using as substrate the fluorogenic compound, 4-methylumbelliferyl $\alpha$-glucoside (Koch-Light), as described previously (6). In some cases, the determination of $\alpha$ glucosidase was made on the homogenate and on the supernatant of the same homogenate after centrifugation.

\section{RESULTS}

Results are summarized in Figures 1 and 2.

(1) Figure 1 shows the results obtained in white blood cells submitted to various types of extraction.

(a) Cells were extracted with water. Alpha-glucosidase activity was measured before and after centrifugation. The percentage of activity in the centrifuged extract, referred to that of the homogenate, was $66 \pm 15 \%$ (column 1). It was 17 and $12 \%$ in two deficient patients, respectively.

(b) Cells were extracted with Triton, $0.2 \%$. After centrifuga- 


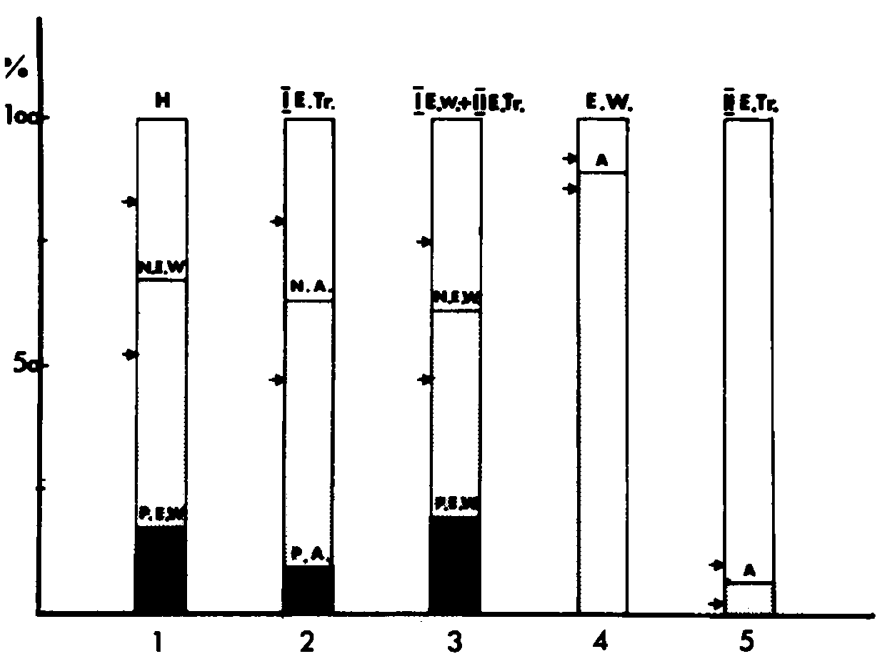

Fig. 1. $\alpha$-Glucosidase activity at $\mathrm{pH} 4$ in various types of extraction. Column $1, \mathrm{H}$, activity in whole homogenate in water without centrifugation. N.E.W., percentage of activity in normal extracts in water. $(N=14)$ after centrifugation, $P . E . W$., percentage of activity in a patient extract in water after centrifugation. Column 2, I.E.Tr, activity in $0.2 \%$ Triton extracts; N.A., percentage of activity due to lysosomal acid maltase obtained after anti acid maltase antiserum $(N=14)$. P.A., patient lysosomal acid maltase activity. Column 3, I.E.W. + II E. Tr, sum of activity extracted first in water and then in $0.2 \%$ Triton; N.E.W., percentage of activity extracted in water $(N=14)$ in normal extracts; $P . E . W$., percentage of activity extracted in water in one patient. Column $4, E$.W., total activity extracted in water (centrifuged extract), $A$, percentage of activity due to lysosomal acid maltase. Column $5, I I E$. $T r$, total activity extracted with $0.2 \%$ Triton after exhaustive washing with water; $A$, percentage of activity due to lysosomal acid maltase. Arrows, limits of standard deviation.

tion, $\alpha$-glucosidase was assayed before and after exposure to anti-acid maltase antiserum. The percentage of acid maltase was $62 \pm 14 \%$ (column 2). When the same procedure was applied to leukocytes of adult patients deficient in acid maltase, the percentage of acid maltase fell to $10 \%$ in both cases.

(c) Cells were extracted with water, centrifuged, and washed with water. After centrifugation, the pellet was extracted with $0.2 \%$ Triton. $\alpha$-Glucosidase activity was determined in both aqueous and Triton extracts. The percentage of activity extracted in water alone (referred to the sum of activity of water and Triton extracts) was $61 \pm 15 \%$ (column 3). It was 19 and $30 \%$ in two deficient patients. From the above results, it appears that the percentage of $\alpha$-glucosidase activity determined in water extracts, referred to total activity of homogenates to the sum of activities in water and Triton, is approximately the same. It is also equal to the percentage of acid maltase as determined immunologically in a Triton extract.

(d) The above conclusions were confirmed by an additional observation: addition of anti-acid maltase antiserum to aqueous extracts and to subsequent Triton extracts showed that their isozyme composition is very different. The aqueous extracts contained nearly only acid maltase (89 $\pm 3 \%$ ). By contrast, the Triton extracts (after exhaustive washing with water) contained practically no acid maltase $(6 \pm 3.5 \%)$, and, therefore, contained nearly only renal maltase because the ubiquitous neutral maltase shows no activity at pH 4.0 (7) (columns 4 and 5).

These experiments demonstrate that the acid maltase is nearly entirely extractable with water with very little contamination by the renal maltase.

In a patient with a late form of $\alpha$-glucosidase deficiency, the percentage of activity in water extracts was $17 \%$ when referred to the whole homogenate and $19 \%$ when referred to the sum of water and subsequent Triton extracts. This is well below the normal percentage although somewhat higher than the residual activity of $10 \%$ obtained with the antiserum. The diagnosis, then, would have been possible without antiserum. It would probably be easier in the infantile form of the deficiency (Pompe's disease) because in this form of the disease no residual activity remains.

(2) Figure 2 shows the percentage of renal maltase in $0.2 \%$ Triton extracts of various types of cells as obtained by the combined action of two antisera directed respectively against acid and renal maltases whose results confirm each other.

(a) In whole white blood cell extracts, the mean percentage was $38 \pm 15$ (column 1), a result which is complementary to that expressed in Figure 1, column 2. In two patients, this percentage rose to $90 \%$ (column 2).

(b) In leukocyte extracts from two patients with chronic myeloid leukemia, the percentage was 18 and $45 \%$ (column 3).

(c) In extracts from three lymphocyte preparations (containing some monocytes), the percentage was close to $5 \%$ (column 4).

(d) No renal maltase could be found in leukocyte extracts from two patients with acute myeloblastic leukemia (column 5) and from two patients with chronic lymphocytic leukemia (column 6).

(e) No renal maltase was found in cell extracts from any of the five tested established lymphoid lines (column 7).

Renal maltase, then, was only found in normal or leukemic granulocytes, provided they be mature. None was found in immature cells (leukemic myeloblasts) and in normal or leukemic lymphocytes.

\section{DISCUSSION}

In a previous paper, we have shown that extracts from human leukocytes contain two types of $\alpha$-glucosidases active at $\mathrm{pH} 4.0$ (7). One is the ubiquitous lysosomal acid glucosidase. The other is immunologically identical to the renal maltase which seems to be present only in kidney and in white blood cells. This isozyme could not be found in other human tissues, including cultured fibroblasts and cultured amniotic cells (J. C. Dreyfus and L. Poenaru, unpublished results). This fact explains that the defi-

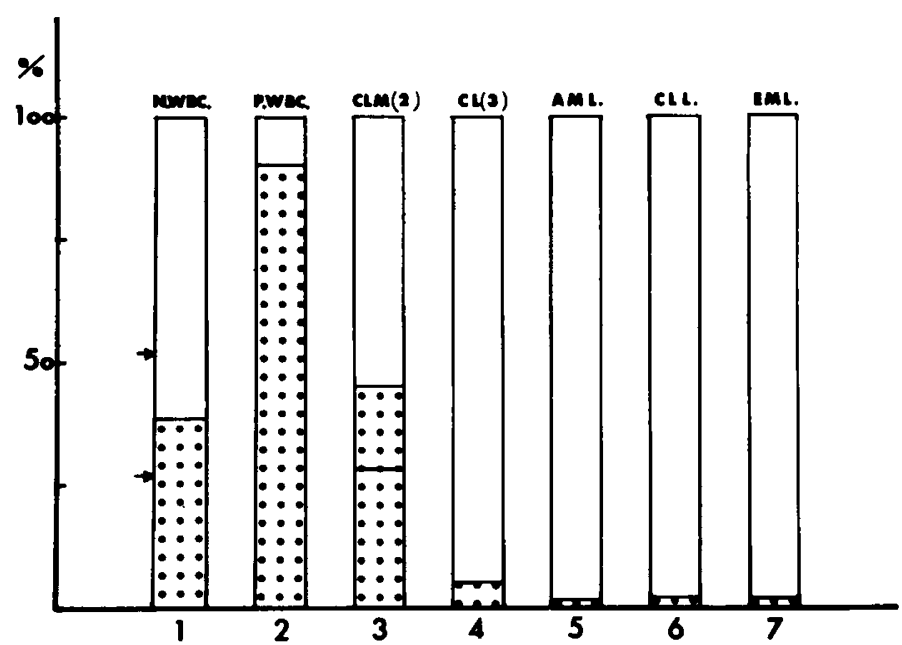

Fig. 2. Percentage of renal maltase in extracts from various types of cells. Dotted area of bars, percentage of renal maltase, referred to the whole activity of the extract taken as $100 \%$. Column 1 , whole, unfractionated normal white blood cells $(N=14)$; column 2, two adult patients with acid $\alpha$-glucosidase deficiency; column 3, two patients with chronic myelocytic leukemia; column 4, normal lymphocytes (three preparations); column 5, two patients with acute myeloblastic leukemia; column 6, two patients with chronic lymphocytic leukemia; column 7 , five different established lymphoid lines. 
ciency of $\alpha$-glucosidase (Pompe's disease) could be reliably ascertained on leukocytes by the use of a specific antiserum.

The present work demonstrates the following facts: (1) lysosomal $\alpha$-glucosidase is readily extracted with distilled water. Very little, if any, remains in the sediment to be extracted with Triton. By contrast, renal maltase remains nearly entirely in the sediment after water extraction and can be extracted with Triton. Addition of antirenal maltase antiserum to aqueous extracts is ineffective, but removes most of the activity of the subsequent Triton extract; (2) comparison of activity of the whole homogenate and of the centrifuged extract (Fig. 1, column 1) shows that the enzyme which is not extracted is nevertheless active in the suspended homogenate. Renal maltase, therefore, is determined along with acid maltase if the water extract is not centrifuged. From the above results, it seems clear that extracting in water and centrifuging provide the best conditions for a specific determination of acid glucosidase, minimizing the contamination by renal maltase. Comparison of extraction with water and Triton gives additional information. The latter results, however, must be regarded with caution, because the amount of renal maltase may be variable. In infants, in particular, when the percentage of lymphocytes is high, renal maltase could be very low. This point requires additional studies; (3) comparison between various types of white cells shows that the presence of renal maltase is specific for adult forms of granulocytes. It is present in normal granulocytes (and perhaps in monocytes) and in myelocytes from chronic myelocytic leukemia, but not in immature myeloblasts from acute myeloblastic leukemia. It is absent or nearly absent from normal lymphocytes, from leukemic lymphocytes, and from all five established lymphoid lines which we have tested.

From a practical point of view, we conclude that when a diagnosis of acid glucosidase deficiency is attempted on extracts from white blood cells, determinations should be made on aqueous, centrifuged extracts, and not on whole homogenates or on cells extracted with detergents. The results would be still more reliable using lymphocytes rather than whole white blood cells. Finally, confirmation by using anti-acid maltase antiserum, if available, would eliminate any risk of error.

\section{REFERENCES AND NOTES}

1. Angelini, C. and Engel, A. G.: Comparative study of acid maltase deficiency. Arch. Neurol., 26: 344 (1972).

2. Bienvenu, J., Carrier, H., Fremon, F., and Mathieu, M.: Hétérogénéité de la glycogénose par déficit en alpha-1,4-glucosidase: étude enzymatique dans trois familles. Clin. Chim. Acta, 84: 277 (1978).

3. Broadhead, D. M., and Butterworth, J.: Pompe's disease: diagnosis in kidney and leucocytes using 4-methylumbelliferyl- $\alpha-D$ glucopyranoside. Clin. Genet., 13: 504 (1978).

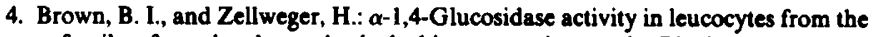
family of two brothers who lack this enzyme in muscle. Biochem. J. 101: 16 (1966).

5. De Burlet, G., and Sudaka, P.: Purification de la maltase neutre rénale humaine. Biochimie (Paris), 58: 621 (1976).

6. Dreyfus, J. C., and Poenaru, L.: Le diagnostic enzymatique des sphingolipidoses. Arch. Franc. Pediatr., 32: 503 (1975).

7. Dreyfus, J. C., and Poenanu, L.: Alpha-glucosidases in white blood cells with reference to the detection of acid $\alpha-1-4$ glucosidase deficiency. Biochem. Biophys. Res. Commun. 85: 615 (1978).

8. Dreyfus, J. C., Poenaru, L., and Lenoir, G.: Activity of lysosomal hydrolases in established lymphoid cell lines. Biomedicine (Paris) (in press).

9. Hudgson, P., Gardner-Medwin, D., and Worsfold, M.: Adult myopathy from glycogen storage disease due to acid maltase deficiency. Brain, 91: 435 (1968).

10. Hui-Jing F., Van Crefeld, S., and Losekoot, G.: Diagnosis of generalized glycogen storage disease (Pompe's disease). J. Pediatr., 63: 984 (1963).

11. Kahn, A., Boivin, P., Viber, M., Cottreau, D., and Dreyfus, J. C.: Posttranslational modifications of human glucose-6-phosphate dehydrogenase. Biochimie, 56: 1395 (1974).

12. Koster, J. F., Slee, R. G., and Hülsmann, W. C.: The use of leucocytes as an aid in the diagnosis of a variant of glycogen storage disease type II (Pompe's disease). Eur. J. Clin. Invest., 2: 467 (1972).

13. Mehler, M., and Di Mauro, S.: Residual acid maltase activity in late onset acid maltase deficiency. Neurology, 27: 178 (1977).

14. Salafsky, I. S., and Nadler, H. L.: Alpha-1,4-glucosidase activity in Pompe's disease. J. Pediatr., 79: 794 (1971).

15. Taniguchi, N., Kato, E., Yoshida, H., Iwaki, S., Ohki, T., and Koizumi, S.: Alpha glucosidase activity in human leucocytes: choice of lymphocytes for the diagnosis of Pompe's disease and the carrier state. Clin. Chim. Acta, 89: 293 (1978).

16. We attest that informed consent was obtained from the persons whose blood samples were used for the present study.

17. Requests for reprints should be addressed to: J. C. Dreyfus, Ph.D., M.D., Institut de Pathologie Moléculaire, INSERM U 129, 24 rue du Faubourg St. Jacques, 75674, Paris CeDEX 14, France.

18. This research was supported in part by grant INSERM ORL 76. 50262.

19. Received for publication May 22, 1979.

20. Accepted for publication July 27, 1979. 